\title{
Properties of an oriented ring of neurons with the FitzHugh-Nagumo model
}

\author{
E. G. Fedorov
}

ITMO University, Kronverkskiy, 49, St. Petersburg, 197101, Russia

FedorovEGe@gmail.com

PACS 87.19.La, 87.18.Sn

DOI 10.17586/2220-8054-2021-12-5-553-562

\begin{abstract}
The transmission of an impulse through a neuron is provided by processes that occur at the nanoscale level. This paper will build a model for an oriented ring of connected neurons. To describe the process of impulse transmission through a neuron, the FitzHugh-Nagumo model is used, which allows one to set a higher abstraction level by simulating an impulse. In this case, when transmitting impulses between neurons, the delay is taken into account. For the constructed model, the dependence of the number of neurons on the dynamics of the network as a whole is studied, and local bifurcations are found. All results are verified numerically. It is shown that the period of self-oscillations of such a network depends on the number of neurons.
\end{abstract}

Keywords: Neural networks, Hopf bifurcations, FitzHugh-Nagumo system, communication delay, stability.

Received: 15 July 2021

Revised: 19 September 2021

\section{Introduction}

The time delay can greatly affect the network of neurons and significantly expand the range of possible behaviors of the network. Because of this, models of networks are actively being investigated, where transmission between neurons occurs with a delay. There are works here on a network of connected neurons with an identical delay [1,2]. And works on different transmission lag times [3-6]. Most often, in such studies, a pair of connected neurons is considered.

The transmission of an impulse through a neuron is provided by processes that occur at the nanoscale level, for example, via the overflow of sodium, potassium, chlorine and calcium ions through ion channels. In this work, we study the effect of the delay in the transmission of an impulse between connected neurons on the dynamics of the system of neurons as a whole. The FitzHugh-Nagumo model will be used to describe one neuron. And the system as a whole will be an oriented ring of neurons connected one after another. In this case, any connection between neurons will be considered with a constant delay. The main goal is to determine the type of behavior depending on the delay time, as well as to test the hypothesis that there is no direct relationship between the number of neurons and the period of self-oscillation of the impulse in the system. For this, in the first part of the work, the analysis of equilibrium states and their stability is carried out. In the second part of the work, the results obtained are verified numerically.

\section{Neuron network}

We will consider a group of neurons, where each neuron can have unidirectional connections with other neurons. That is, the axon of a neuron can be called its output, and for another neuron it will be an input (through a bunch of synapses and dendrites). To describe one neuron, will use the FitzHugh-Nagumo model [7-9]:

$$
\left\{\begin{array}{l}
\dot{u}=-a u+(a+1) u^{2}-u^{3}-v+I \\
\dot{v}=b u-\gamma v
\end{array}\right.
$$

where $u(t)$ corresponds to the potential of the neuron at time $t$, and $v(t)$ is the function of the internal state at time $t$, $a, b, \gamma$ are positive constant parameters of the neuron, and $I$ is the external current to the neuron.

As an external current $I$ for a neuron, we will consider the total impact of other neurons, and the output will be the value of the potential of a neuron with a delay $\tau$ (delay for the transmission of an impulse through an axon). In this case, the effect between neurons will be sigmoidal (that is, it does not depend on the postsynaptic neuron). Thus, the model of the $i$-th neuron will be as follows:

$$
\left\{\begin{array}{l}
\dot{u}_{i}=-a u_{i}+(a+1) u_{i}^{2}-u_{i}^{3}-v_{i}+\sum_{j=1, j \neq i}^{n} c \tanh \left(u_{j}^{\tau}\right) \\
\dot{v}_{i}=b u_{i}-\gamma v_{i}
\end{array}\right.
$$




\subsection{Ring of connected neurons}

In this section, we will consider a specific network of neurons in the form of an oriented ring. That is, the input of the neuron $i$ will be the output of the neuron $i-1(i \geq 2)$, and the input of the neuron 1 will be the output of the neuron $n$. Moreover, all neurons are identical in their parameters. A schematic representation of the considered network in Fig. 1. We will write a model of the considered network of neurons:

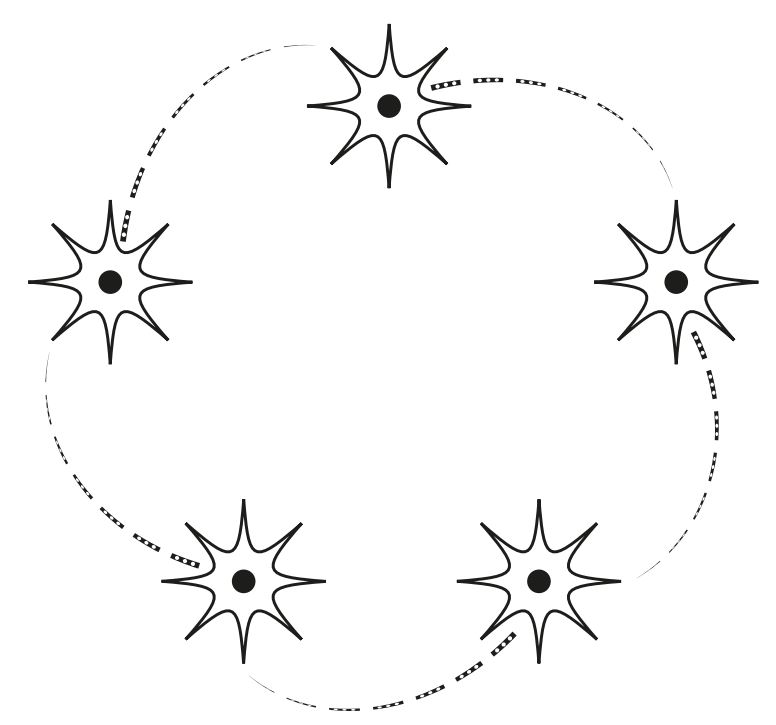

FIG. 1. Schematic representation of an oriented ring of connected neurons

$$
\left\{\begin{aligned}
\dot{u}_{1} & =-a u_{1}+(a+1) u_{1}^{2}-u_{1}^{3}-v_{1}+c \tanh \left(u_{n}^{\tau}\right), \\
\dot{v}_{1} & =b u_{1}-\gamma v_{1}, \\
\dot{u}_{2} & =-a u_{2}+(a+1) u_{2}^{2}-u_{2}^{3}-v_{2}+c \tanh \left(u_{1}^{\tau}\right), \\
\dot{v}_{2} & =b u_{2}-\gamma v_{2}, \\
& \ldots \\
\dot{u}_{n} & =-a u_{n}+(a+1) u_{n}^{2}-u_{n}^{3}-v_{n}+c \tanh \left(u_{n-1}^{\tau}\right), \\
\dot{v}_{n} & =b u_{n}-\gamma v_{n},
\end{aligned}\right.
$$

where $u_{i}(t)$ corresponds to the potential of the $i$-th neuron at time $t$, and $v_{i}(t)$ is the function of the internal state at time $t, a, b, \gamma$ are positive constant parameters of neurons, $c$ positive constant, connection strength, $u_{i}^{\tau}(t)=u_{i}(t-\tau)$, $i=1,2, \ldots n$.

2.1.1. Find equilibrium states. To search for equilibrium states of the system, it is necessary to solve:

$$
\begin{aligned}
& \left\{\begin{aligned}
0 & =-a u_{1}+(a+1) u_{1}^{2}-u_{1}^{3}-v_{1}+c \tanh \left(u_{n}\right), \\
0 & =b u_{1}-\gamma v_{1} \\
0 & =-a u_{2}+(a+1) u_{2}^{2}-u_{2}^{3}-v_{2}+c \tanh \left(u_{1}\right), \\
0 & =b u_{2}-\gamma v_{2}, \\
& \cdots \\
0 & =-a u_{n}+(a+1) u_{n}^{2}-u_{n}^{3}-v_{n}+c \tanh \left(u_{n-1}\right), \\
0 & =b u_{n}-\gamma v_{n},
\end{aligned}\right. \\
& \left\{\begin{aligned}
v_{i} & =\frac{b}{\gamma} u_{i}, \quad i \in[1 \ldots n], \\
c \tanh \left(u_{i-1}\right) & =u_{i}^{3}-(a+1) u_{i}^{2}+\left(a+\frac{b}{\gamma}\right) u_{i}, \quad i \in[2 \ldots n], \\
c \tanh \left(u_{n}\right) & =u_{1}^{3}-(a+1) u_{1}^{2}+\left(a+\frac{b}{\gamma}\right) u_{1} .
\end{aligned}\right.
\end{aligned}
$$


Some of the equations in the system can be represented as:

$$
\left\{\begin{aligned}
f\left(u_{i-1}\right) & =g\left(u_{i}\right), \\
f\left(u_{n}\right) & =g\left(u_{1}\right),
\end{aligned}\right.
$$

where $f(u)=c \tanh (u), g(u)=u^{3}-(a+1) u^{2}+\left(a+\frac{b}{\gamma}\right) u$. Let $\frac{b}{\gamma} \geq \frac{1}{3}\left(a^{2}-a+1\right)$. This assumption fits the main parameters of the neurons under consideration $[2,10]$, where $a<1$, and $b$ roughly the same $\gamma$. Throughout the rest of this section, we will assume everywhere that the assumption is fulfilled.

Proposition 1. In the described system, the equilibrium states of neurons are the same, that is $u_{i}=u_{j}, v_{i}=v_{j} \forall i, j$.

For the proof, we show that the function $g(x)$ will be increasing. Derivative $g(x): g^{\prime}(u)=3 u^{2}-2(a+1) u+a+\frac{b}{\gamma}$. Then, if $\frac{b}{\gamma} \geq \frac{1}{3}\left(a^{2}-a+1\right)$, then $(a+1)^{2}-3\left(a+\frac{b}{\gamma}\right) \leq 0$, which coincides with the determinant $g(u) / 2$. Hence, $g(u)$ is an increasing function (the leading coefficient is positive). Now, for the system (6), the functions $f(u)$ and $g(u)$ are increasing, which means that the assumption that some two connected neurons have different values of the equilibrium state potential leads to contradiction. That is, $u_{i}=u_{j} \forall i, j$, hence $v_{i}=v_{j} \forall i, j$.

Now, the system (5) can be reduced to an equivalent:

$$
\left\{\begin{aligned}
v_{i} & =\frac{b}{\gamma} u_{i}, \quad i \in[1 \ldots n], \\
u_{i} & =u_{1}, \quad i \in[2 \ldots n], \\
c \tanh \left(u_{1}\right) & =u_{1}^{3}-(a+1) u_{1}^{2}+\left(a+\frac{b}{\gamma}\right) u_{1} .
\end{aligned}\right.
$$

Now the search for equilibrium states has been reduced to finding the roots of the equation $c \tanh \left(u_{1}\right)=u_{1}^{3}-$ $(a+1) u_{1}^{2}+\left(a+\frac{b}{\gamma}\right) u_{1}$. Note that $u_{1}=0$ is always a root. The right side of the equation has only one inflection point and it is located on the right semiaxis. Now let's highlight a few cases:

1) $a+\frac{b}{\gamma}>c$, then the considered equation has no negative solutions, but positive solutions can be 0,1 or 2 (as $c$ grows, the number of roots grows from 0 to 2,1 root at the only critical value of $c$ ).

2) $a+\frac{b}{\gamma}<c$, then the considered equation has only one negative and one positive solution.

2.1.2. Define the stability of equilibrium states. To determine the stability of the equilibrium states of the system of neurons under consideration, we linearize the system in the general equilibrium state $u_{i}=u_{1}, v_{i}=v_{1}, i \in[2 \ldots n]$. For this, we rewrite the system (3) as $\dot{U}(t)=A U(t)+B U(t-\tau)$, where $U(t)=\left(u_{1}(t), v_{1}(t), \ldots, u_{n}(t), v_{n}(t)\right)^{T}:$

$$
A=\left(\begin{array}{ccccccc}
-\alpha & -1 & 0 & 0 & \cdots & 0 & 0 \\
b & -\gamma & 0 & 0 & \cdots & 0 & 0 \\
0 & 0 & -\alpha & -1 & \cdots & 0 & 0 \\
0 & 0 & b & -\gamma & \cdots & 0 & 0 \\
& & & \cdots & & & \\
0 & 0 & 0 & 0 & \cdots & -\alpha & -1 \\
0 & 0 & 0 & 0 & \cdots & b & -\gamma
\end{array}\right), \quad B=\left(\begin{array}{ccccccc}
0 & 0 & \cdots & 0 & 0 & c^{\prime} & 0 \\
0 & 0 & \cdots & 0 & 0 & 0 & 0 \\
c^{\prime} & 0 & \cdots & 0 & 0 & 0 & 0 \\
0 & 0 & \cdots & 0 & 0 & 0 & 0 \\
& & & \cdots & & & \\
0 & 0 & \cdots & c^{\prime} & 0 & 0 & 0 \\
0 & 0 & \cdots & 0 & 0 & 0 & 0
\end{array}\right),
$$

where $\alpha=3 u_{1}^{2}-2 u_{1}(a+1)+a, c^{\prime}=\frac{c}{\cosh ^{2}\left(u_{1}\right)}$.

Next, we find solutions to the characteristic equation $\operatorname{det}\left(A-\lambda I+B e^{-\lambda \tau}\right)=0$ to determine stability:

$$
\left|\begin{array}{ccccccccc}
-\alpha-\lambda & -1 & 0 & 0 & \cdots & 0 & 0 & c^{\prime} e^{-\lambda \tau} & 0 \\
b & -\gamma-\lambda & 0 & 0 & \cdots & 0 & 0 & 0 & 0 \\
c^{\prime} e^{-\lambda \tau} & 0 & -\alpha-\lambda & -1 & \cdots & 0 & 0 & 0 & 0 \\
0 & 0 & b & -\gamma-\lambda & \cdots & 0 & 0 & 0 & 0 \\
& & & & \cdots & & & & \\
0 & 0 & 0 & 0 & \cdots & c^{\prime} e^{-\lambda \tau} & 0 & -\alpha-\lambda & -1 \\
0 & 0 & 0 & 0 & \cdots & 0 & 0 & b & -\gamma-\lambda
\end{array}\right|=0
$$


To find the determinant, let's write it down on the top line. The first two elements will reduce the problem to a similar one with a lower dimension and this part of the determinant is equal to $((-\alpha-\lambda)(-\gamma-\lambda)+b)^{n}$. The third element in the top line is unique and sets a set of elements in the matrix and the part corresponding to it is equal to $-\left(c^{\prime} e^{-\lambda \tau}(-\gamma-\lambda)\right)^{n}$. As a result:

$$
\begin{gathered}
((-\alpha-\lambda)(-\gamma-\lambda)+b)^{n}-\left(c^{\prime} e^{-\lambda \tau}(-\gamma-\lambda)\right)^{n}=0 \\
(\alpha+\lambda)(\gamma+\lambda)+b=c^{\prime} e^{-\lambda \tau+\frac{2 \pi k}{n} i}(\gamma+\lambda), \quad k \in[0 \ldots n-1] .
\end{gathered}
$$

To determine stability in the system in the absence of delay, take $\tau=0$. Then:

$$
\lambda^{2}+\lambda\left(\alpha+\gamma-c^{\prime} e^{\frac{2 \pi k}{n} i}\right)+\alpha \gamma+b-c^{\prime} \gamma e^{\frac{2 \pi k}{n} i}=0, \quad k \in[0 \ldots n-1]
$$

For even odd $n$, these equations can be split into pairs $(k ;-k)$ and there remains one equation for $k=0$. For even $n$ these equations can be split into pairs $(k ;-k)$ and there will remain two equations for $k=0$ and $k=\frac{n}{2}$. Next, we define the stability of the selected groups.

For $k=0$ :

$$
\lambda^{2}+\lambda\left(\alpha+\gamma-c^{\prime}\right)+\alpha \gamma+b-c^{\prime} \gamma=0
$$

This pair of roots will be stable if and only if:

$$
\left\{\begin{array}{c}
\alpha+\gamma-c^{\prime}>0 \\
\alpha+\frac{b}{\gamma}-c^{\prime}>0
\end{array}\right.
$$

That is $\alpha+\min \left(\gamma, \frac{b}{\gamma}\right)>c^{\prime}$. And, for the zero state of equilibrium:

$$
a+\min \left(\gamma, \frac{b}{\gamma}\right)>c
$$

For $k=\frac{n}{2} \quad(n: 2):$

This pair of roots will be stable if and only if:

$$
\lambda^{2}+\lambda\left(\alpha+\gamma+c^{\prime}\right)+\alpha \gamma+b+c^{\prime} \gamma=0
$$

$$
\left\{\begin{array}{l}
\alpha+\gamma+c^{\prime}>0 \\
\alpha+\frac{b}{\gamma}+c^{\prime}>0
\end{array}\right.
$$

That is $\alpha+\min \left(\gamma, \frac{b}{\gamma}\right)>-c^{\prime}$. For a zero equilibrium state, this condition is automatically satisfied, since $c>0$.

For couple $(k ;-k)\left(k \neq 0, k \neq \frac{n}{2}\right)$ :

$$
\begin{gathered}
\left(\lambda^{2}+\lambda\left(\alpha+\gamma-c^{\prime} e^{-\frac{2 \pi k}{n} i}\right)+\alpha \gamma+b-c^{\prime} \gamma e^{\frac{-2 \pi k}{n} i}\right) \times \\
\left(\lambda^{2}+\lambda\left(\alpha+\gamma-c^{\prime} e^{\frac{2 \pi k}{n} i}\right)+\alpha \gamma+b-c^{\prime} \gamma e^{\frac{2 \pi k}{n} i}\right)=0, \\
\left(\lambda^{2}+\lambda(\alpha+\gamma)+\alpha \gamma+b\right)^{2}-2\left(\lambda^{2}+\lambda(\alpha+\gamma)+\alpha \gamma+b\right) c^{\prime}(\gamma+\lambda) \cos \frac{2 \pi k}{n}+c^{\prime 2}(\gamma+\lambda)^{2}=0, \\
\lambda^{4}+2\left(\alpha+\gamma-c^{\prime} \cos \frac{2 \pi k}{n}\right) \lambda^{3}+\left((\alpha+\gamma)^{2}+2(\alpha \gamma+b)-2(\alpha+2 \gamma) c^{\prime} \cos \frac{2 \pi k}{n}+c^{2}\right) \lambda^{2}+ \\
2\left((\alpha+\gamma)(\alpha \gamma+b)-(\alpha+\gamma) c^{\prime} \gamma \cos \frac{2 \pi k}{n}-(\alpha \gamma+b) c^{\prime} \cos \frac{2 \pi k}{n}+c^{\prime 2} \gamma\right) \lambda+ \\
(\alpha \gamma+b)^{2}-2(\alpha \gamma+b) c^{\prime} \gamma \cos \frac{2 \pi k}{n}+c^{\prime 2} \gamma^{2}=0 .
\end{gathered}
$$

An equation of the 4-th degree with real coefficients is obtained, its stability can be checked by the Hurwitz or Mikhailov criterion.

Now, we will find candidates for bifurcation from stable to unstable equilibrium or vice versa (when there is a purely imaginary eigenvalue). To do this, we take $\lambda=i \omega$ :

$$
\begin{gathered}
(\alpha+i \omega)(\gamma+i \omega)+b=c^{\prime} e^{-i \omega \tau+\frac{2 \pi k}{n} i}(\gamma+i \omega), \\
\left\{\begin{array}{c}
\alpha \gamma-\omega^{2}+b+i \omega(\alpha+\gamma)=c^{\prime}\left(\gamma \cos \tau^{\prime}+\omega \sin \tau^{\prime}+i\left(\omega \cos \tau^{\prime}-\gamma \sin \tau^{\prime}\right)\right) \\
\tau^{\prime}=\omega \tau-\frac{2 \pi k}{n},
\end{array}\right.
\end{gathered}
$$




$$
\begin{gathered}
\left\{\begin{aligned}
\alpha \gamma-\omega^{2}+b & =c^{\prime}\left(\gamma \cos \tau^{\prime}+\omega \sin \tau^{\prime}\right), \\
\omega(\alpha+\gamma) & =c^{\prime}\left(\omega \cos \tau^{\prime}-\gamma \sin \tau^{\prime}\right), \\
\tau^{\prime} & =\omega \tau-\frac{2 \pi k}{n},
\end{aligned}\right. \\
\left\{\begin{aligned}
c^{\prime}\left(\omega^{2}+\gamma^{2}\right) \cos \tau^{\prime} & =\alpha\left(\omega^{2}+\gamma^{2}\right)+b \gamma, \\
c^{\prime}\left(\omega^{2}+\gamma^{2}\right) \sin \tau^{\prime} & =-\omega\left(\omega^{2}+\gamma^{2}\right)+b \omega, \\
\tau^{\prime} & =\omega \tau-\frac{2 \pi k}{n},
\end{aligned}\right.
\end{gathered}
$$

let $\tau^{\prime} \neq \frac{\pi}{2}+\pi k, k \in \mathbb{Z}$, then:

$$
\begin{aligned}
& \left\{\begin{array}{c}
\tan \left(\omega \tau-\frac{2 \pi k}{n}\right)=\frac{-\omega\left(\omega^{2}+\gamma^{2}\right)+b \omega}{\alpha\left(\omega^{2}+\gamma^{2}\right)+b \gamma} \\
\operatorname{sign}\left(\cos \left(\omega \tau-\frac{2 \pi k}{n}\right)\right)=\operatorname{sign}\left(\alpha\left(\omega^{2}+\gamma^{2}\right)+b \gamma\right) \\
c^{\prime 2}\left(\omega^{2}+\gamma^{2}\right)^{2}=\left(\alpha\left(\omega^{2}+\gamma^{2}\right)+b \gamma\right)^{2}+\left(-\omega\left(\omega^{2}+\gamma^{2}\right)+b \omega\right)^{2}
\end{array}\right. \\
& \left\{\begin{array}{c}
\tan \left(\omega \tau-\frac{2 \pi k}{n}\right)=\frac{-\omega\left(\omega^{2}+\gamma^{2}\right)+b \omega}{\alpha\left(\omega^{2}+\gamma^{2}\right)+b \gamma} \\
\operatorname{sign}\left(\cos \left(\omega \tau-\frac{2 \pi k}{n}\right)\right)=\operatorname{sign}\left(\alpha\left(\omega^{2}+\gamma^{2}\right)+b \gamma\right) \\
c^{\prime 2}\left(\omega^{2}+\gamma^{2}\right)=\alpha^{2}\left(\omega^{2}+\gamma^{2}\right)+2 b \alpha \gamma+b^{2}+\omega^{2}\left(\omega^{2}+\gamma^{2}\right)-2 b \omega^{2}
\end{array}\right. \\
& \left\{\begin{array}{c}
\tan \left(\omega \tau-\frac{2 \pi k}{n}\right)=\frac{-\omega\left(\omega^{2}+\gamma^{2}\right)+b \omega}{\alpha\left(\omega^{2}+\gamma^{2}\right)+b \gamma} \\
\operatorname{sign}\left(\cos \left(\omega \tau-\frac{2 \pi k}{n}\right)\right)=\operatorname{sign}\left(\alpha\left(\omega^{2}+\gamma^{2}\right)+b \gamma\right) \\
\omega^{4}+\left(\alpha^{2}+\gamma^{2}-c^{\prime 2}-2 b\right) \omega^{2}+\alpha^{2} \gamma^{2}+2 b \alpha \gamma+b^{2}-c^{\prime 2} \gamma^{2}=0
\end{array}\right.
\end{aligned}
$$

This means that the system can have 0,1 , or 2 series of decisions. Under the condition $\alpha\left(\omega^{2}+\gamma^{2}\right)+b \gamma>0$ (which is always true for the zero equilibrium state), we obtain (if the condition is not true, then the series for $\tau^{\prime}$ must be offset by $\pi)$ :

$$
\begin{aligned}
& \left\{\begin{array}{c}
\omega=\left(\frac{1}{2}\left(-\left(\alpha^{2}+\gamma^{2}-c^{\prime 2}-2 b\right) \pm \sqrt{\left(\alpha^{2}-\gamma^{2}-c^{\prime 2}\right)^{2}-4 b\left((\alpha+\gamma)^{2}-c^{\prime 2}\right)}\right)\right)^{\frac{1}{2}} \\
\tau=\frac{1}{\omega} \arctan \left(\frac{-\omega\left(\omega^{2}+\gamma^{2}\right)+b \omega}{\alpha\left(\omega^{2}+\gamma^{2}\right)+b \gamma}\right)+\frac{2 \pi m}{\omega}+\frac{2 \pi k}{n \omega}, \quad k, m \in \mathbb{Z},
\end{array}\right. \\
& \left\{\begin{array}{c}
\omega=\left(\frac{1}{2}\left(-\left(\alpha^{2}+\gamma^{2}-c^{\prime 2}-2 b\right) \pm \sqrt{\left(\alpha^{2}-\gamma^{2}-c^{\prime 2}\right)^{2}-4 b\left((\alpha+\gamma)^{2}-c^{\prime 2}\right)}\right)\right)^{\frac{1}{2}}, \\
\tau=\frac{1}{\omega} \arctan \left(\frac{-\omega\left(\omega^{2}+\gamma^{2}\right)+b \omega}{\alpha\left(\omega^{2}+\gamma^{2}\right)+b \gamma}\right)+\frac{2 \pi k}{n \omega}, \quad k \in \mathbb{Z} .
\end{array}\right.
\end{aligned}
$$

Next, we define the conditions for the presence of candidates for a local bifurcation. That is, the conditions under which (27) has a real or positive root for $\omega^{2}$. If:

1) $c^{\prime}>\left|\alpha+\frac{b}{\gamma}\right|$, in for $\omega=0$ (27) is negative, therefore, with respect to $\omega^{2}$ there is exactly one positive solution.

2) $c^{\prime}<\left|\alpha+\frac{b}{\gamma}\right|,(27)$ will be positive at 0 , then it is necessary and sufficient that the roots are (the discriminant is positive) and the coefficient at $\omega^{2}$ was negative. That is:

$$
\begin{gathered}
\left\{\begin{array}{c}
\left(\alpha^{2}+\gamma^{2}-c^{2}-2 b\right)^{2}-4\left(\alpha^{2} \gamma^{2}+2 b \alpha \gamma+b^{2}-c^{2} \gamma^{2}\right)>0, \\
\alpha^{2}+\gamma^{2}-c^{2}-2 b<0,
\end{array}\right. \\
\left\{\begin{array}{c}
c^{\prime 4}-2\left(\alpha^{2}-\gamma^{2}-2 b\right) c^{\prime 2}+\alpha^{4}-2 \alpha^{2} \gamma^{2}+\gamma^{4}-4 \alpha^{2} b-8 \alpha b \gamma-4 b \gamma^{2}>0, \\
c^{\prime 2}>\alpha^{2}+\gamma^{2}-2 b .
\end{array}\right.
\end{gathered}
$$

For $2 \alpha b \gamma+2 b \gamma^{2}+b^{2}<0$, the system is equivalent to $c^{\prime 2}>\alpha^{2}+\gamma^{2}-2 b$, otherwise:

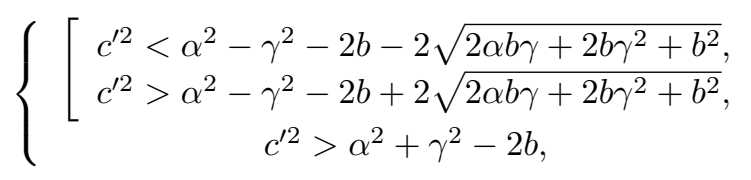

$$
\begin{aligned}
& c^{\prime 2}>\alpha^{2}-\gamma^{2}-2 b+2 \sqrt{2 \alpha b \gamma+2 b \gamma^{2}+b^{2}} .
\end{aligned}
$$


Next, it is necessary to determine for each candidate for bifurcation its effect on stability (increases or decreases the number of eigenvalues with a positive real part). Let $\tau_{0}$ be a candidate for bifurcation, $\omega_{0}, \lambda_{0}=i \omega_{0}$ correspond to it. Define the sign of the expression $\left.\frac{\partial \Re(\lambda)}{\partial \tau}\right|_{\tau=\tau_{0}}$, where $\Re(x)$ is the real part of the number $x, D$ - characteristic equation (10):

where

$$
\left.\frac{\partial \Re(\lambda)}{\partial \tau}\right|_{\tau=\tau_{0}}=-\left.\Re\left(\frac{\partial D}{\partial \tau} / \frac{\partial D}{\partial \lambda}\right)\right|_{\tau=\tau_{0}}=-\Re\left(\frac{n\left(c^{\prime} e^{-\lambda_{0} \tau_{0}}\left(\gamma+\lambda_{0}\right)\right)^{n} \lambda_{0}}{d_{1}}\right),
$$

$$
d_{1}=n\left(\left(\alpha+\lambda_{0}\right)\left(\gamma+\lambda_{0}\right)+b\right)^{n-1}\left(\gamma+\alpha+2 \lambda_{0}\right)-n c^{\prime n} e^{-n \lambda_{0} \tau_{0}}\left(\gamma+\lambda_{0}\right)^{n-1}\left(-\tau_{0}\left(\gamma+\lambda_{0}\right)+1\right) .
$$

Since $\tau_{0}$ and $\lambda_{0}$ satisfy the characteristic equation, then

$$
\left(c^{\prime} e^{-\lambda_{0} \tau_{0}}\left(-\gamma-\lambda_{0}\right)\right)^{n}=\left(\left(-\alpha-\lambda_{0}\right)\left(-\gamma-\lambda_{0}\right)+b\right)^{n},
$$

then

$$
\left.\frac{\partial \Re(\lambda)}{\partial \tau}\right|_{\tau=\tau_{0}}=-\Re\left(\frac{\left(\left(\alpha+\lambda_{0}\right)\left(\gamma+\lambda_{0}\right)+b\right)^{n} \lambda_{0}}{d_{2}}\right)=-\Re\left(\frac{\left(\left(\alpha+\lambda_{0}\right)\left(\gamma+\lambda_{0}\right)+b\right) \lambda_{0}\left(\gamma+\lambda_{0}\right)}{d_{3}}\right),
$$

where

$$
\begin{aligned}
& d_{2}=\left(\left(\alpha+\lambda_{0}\right)\left(\gamma+\lambda_{0}\right)+b\right)^{n-1}\left(\gamma+\alpha+2 \lambda_{0}\right)-\left(\left(\alpha+\lambda_{0}\right)\left(\gamma+\lambda_{0}\right)+b\right)^{n}\left(-\tau_{0}+\frac{1}{\gamma+\lambda_{0}}\right), \\
& d_{3}=\tau_{0} \lambda_{0}^{3}+\left(\alpha \tau_{0}+2 \gamma \tau_{0}+1\right) \lambda_{0}^{2}+\left(2 \alpha \gamma \tau_{0}+\gamma^{2} \tau_{0}+b \tau_{0}+2 \gamma\right) \lambda_{0}++\alpha \gamma^{2} \tau_{0}+b \gamma \tau_{0}+\gamma^{2}-b .
\end{aligned}
$$

Multiply in (37) the numerator and denominator by the conjugate of $d_{3}$ and multiply the whole expression by the square of the modulus $d_{3}$ :

$$
\operatorname{sign}\left(\left.\frac{\partial \Re(\lambda)}{\partial \tau}\right|_{\tau=\tau_{0}}\right)=\operatorname{sign}\left(\left(\omega_{0}^{2}+\gamma^{2}\right)^{2}-\left(b^{2}+2 \alpha b \gamma+2 b \gamma^{2}\right)\right) .
$$

Now, we can see that the condition for increasing/decreasing the number of roots with a positive real part does not depend on a specific representative of the $\tau$ series, but is a property of the entire series or depends only on $\omega$. $\omega_{0}$ is the root of (27):

in another view:

$$
\omega_{0}^{4}+\left(\alpha^{2}+\gamma^{2}-c^{2}-2 b\right) \omega_{0}^{2}+\alpha^{2} \gamma^{2}+2 b \alpha \gamma+b^{2}-c^{\prime 2} \gamma^{2}=0
$$

$$
\left(\omega_{0}^{2}+\gamma^{2}\right)^{2}+\left(\alpha^{2}-c^{2}-\gamma^{2}-2 b\right)\left(\omega_{0}^{2}+\gamma^{2}\right)+b^{2}+2 \alpha b \gamma+2 b \gamma^{2}=0
$$

Lemma 2. Let $x^{2}+a x+b=0$ have two real roots $x_{1}, x_{2}\left(x_{1}<x_{2}\right)$ and $b>0$, then $x_{1}^{2}-b$ and $x_{2}^{2}-b$ will have different signs. And if $a>0$, then $x_{2}^{2}-b<0$, and if $a<0$, then $x_{1}^{2}-b<0$.

Let $x^{2}+a x+b=0$ have two real roots $x_{1}, x_{2}\left(x_{1}<x_{2}\right)$ and $b<0$, then $x_{1}^{2}-b$ and $x_{2}^{2}-b$ will always be positive.

Substitute the values of the roots in $x^{2}-b$ :

$$
\left(\frac{\left(-a \pm \sqrt{a^{2}-4 b}\right)}{2}\right)^{2}-b=\frac{a^{2}-4 b \mp a \sqrt{a^{2}-4 b}}{2},
$$

then $\operatorname{sign}\left(x^{2}-b\right)=\operatorname{sign}\left(\sqrt{a^{2}-4 b} \mp a\right)$. This implies the assertions of the lemma.

As previously received, if $\left|\alpha+\frac{b}{\gamma}\right|<c^{\prime}$, that is, one solution (27) for $\omega$ (up to sign). $\left|\alpha+\frac{b}{\gamma}\right|<c^{\prime}$, so, $\alpha<c^{\prime}$, and therefore $\alpha^{2}-c^{2}-\gamma^{2}-2 b<0$. That is, among the two roots (27) for $\omega^{2}$ ours is larger in absolute value. If $\left|\alpha+\frac{b}{\gamma}\right|>c^{\prime}$, then $\alpha^{2}+\gamma^{2}-c^{2}-2 b<0$, hence $\alpha^{2}-\gamma^{2}-c^{\prime 2}-2 b<0$.

Applying lemma (2), the expression for $\operatorname{sign}\left(\left.\frac{\partial \Re(\lambda)}{\partial \tau}\right|_{\tau=\tau_{0}}\right)$, the inequalities obtained above for $\omega_{0}$ and the equation (let there be at least one solution for $\omega_{0}$ ) obtain that $\operatorname{sign}\left(\left.\frac{\partial \Re(\lambda)}{\partial \tau}\right|_{\tau=\tau_{0}}\right)=-1$, if and only if there are two roots (up to a sign) for $\omega$ and $b^{2}+2 \alpha b \gamma+2 b \gamma^{2}>0$ for all $\tau$, corresponding to a smaller $\omega$ (larger period of the series). 
Then, we can conclude that:

1) If $c>a+\frac{b}{\gamma}$, that is, there are three equilibrium states $(u<0, u=0, u>0)$, while the zero equilibrium state will be unstable for $\tau=0$, and will remain unstable with increasing $\tau$ (there is one series of values that changes the number of roots with a positive real part, but it only increases this number).

2) If $c<a+\frac{b}{\gamma}$, that is, either one equilibrium state $(u=0)$, or three $(u=0, u>0, u>0)$.

For equilibrium states, the following results were obtained:

1) If $c^{\prime}>\alpha+\gamma$ or $c^{\prime}>\alpha+\frac{b}{\gamma}$, then the equilibrium state is unstable at $\tau=0$. If $c^{\prime}<\alpha+\gamma$ and $c^{\prime}<\alpha+\frac{b}{\gamma}$, then it is asymptotically stable.

2) If $c^{\prime}>\left|\alpha+\frac{b}{\gamma}\right|$, then (the equilibrium state is unstable at $\tau=0$ ) the equilibrium state will be unstable for any $\tau$ (there is one series of values, which changes the number of roots with a positive real part, but it only increases this number).

3) Otherwise, if $2 \alpha \gamma+2 \gamma^{2}+b<0$ and $c^{2}>\alpha^{2}+\gamma^{2}-2 b$, that is, two series of changing the number of roots with positive real part, both of which increase this number. If $2 \alpha \gamma+2 \gamma^{2}+b>0$ and $c^{\prime 2}>\alpha^{2}-\gamma^{2}-2 b+$ $2 \sqrt{2 \alpha b \gamma+2 b \gamma^{2}+b^{2}}$, that is, two series of changing the number of roots with a positive real part, one of which decreases the number of roots, the other increases (the one with a shorter period). Otherwise, there are no succession series, the asymptotic stability of the equilibrium state does not depend on $\tau$.

As a special case, for the zero equilibrium state:

1) If $c>a+\frac{b}{\gamma}$, then the equilibrium state will be unstable for any $\tau$ and there are two other equilibrium states.

2) If $a+\frac{b}{\gamma}>c>a+\gamma$, then the equilibrium state is unstable at $\tau=0$ and there are two series of changing the number of roots with a positive real part, one of which decreases the number roots, the other increases.

3) If $c<a+\gamma$, then the equilibrium state is asymptotically stable for $\tau=0$, for $c^{2}<a^{2}-\gamma^{2}-2 b+$ $2 \sqrt{2 a b \gamma+2 b \gamma^{2}+b^{2}}$ it will remain so for any $\tau$, otherwise there are two series of changing the number of roots with a positive real part, one of which decreases the number of roots, the other increases.

In the results obtained, it can be noted that the number and the states of equilibrium themselves do not depend on the number of neurons in the ring. Also, the main criteria for determining the stability of a series of candidates do not depend on the number of neurons. Only the period in this series depends: the period is equal to $\frac{2 \pi}{n \omega}$. Thus, the direct relationship between the number of neurons in the ring and the pulse propagation period was not confirmed analytically.

2.1.3. Numerical verification of conclusion. Let us introduce several definitions: a sequence of the first kind is a sequence $\left\{\tau_{k}\right\}$ such that for the characteristic equation (10) passing through the value of this sequence leads to an increase by two the number of roots with a positive real part. Similarly, introduce a sequence of the second kind - one that reduces by two the number of roots with a positive real part.

To obtain numerical solutions of the system, the Wolfram Mathematica package and the NDSolve method were used. The initial values were zero functions and a jump at the time $t=0$ for the first neuron by 0.01 . That is, $u_{1}(t \mid t<0)=v_{1}(t \mid t \leq 0)=0, u_{1}(0)=0.01, u_{i}(t \mid t \leq 0)=v_{i}(t \mid t \leq 0)=0, i \in[2, \ldots n]$.

Consider the following values of neuron parameters: $a=0.15, b=\gamma=0.02, c=0.18$.

The value $c=0.18$ lies between the value $c=0.890512$, which separates one state of equilibrium from three, and between $c=a+\gamma=0.17$. That is, from the results obtained earlier, we can conclude that, regardless of the number of neurons, there is one equilibrium state $(u=0)$, which is unstable at $\tau=0$. For this state of equilibrium, there is a series of both the first and second kind.

Next, we consider the different number of neurons in the ring:

1) A ring of two neurons. Then two series of candidates are $\tau_{1, k} \approx 1.70691+25.715009 k$ of the second kind, and $\tau_{2, k} \approx 14.431569+16.895513 k$ of the first kind. Then, for this unique equilibrium state, we obtain four Hopf bifurcations: at $\tau \approx 1.70691-$ a transition from an unstable equilibrium to an asymptotically stable one (Fig. 2, 3), then for $\tau \approx 14.431569, \tau \approx 27.42192$ and $\tau \approx 31.327082$ (Fig. 4, 5).

2) A ring of three neurons. Then two series of candidates are $\tau_{1, k} \approx 1.70691+17.143339 k$ of the second kind, and $\tau_{2, k} \approx 8.799731+11.263675 k$ of the first kind. Then, for this unique equilibrium state, we obtain four Hopf bifurcations: at $\tau \approx 1.70691-$ a transition from an unstable equilibrium to an asymptotically stable one (Fig. 6, 7), then for $\tau \approx 8.799731, \tau \approx 18.850249$ and $\tau \approx 20.063406$ (Fig. 8, 9). 


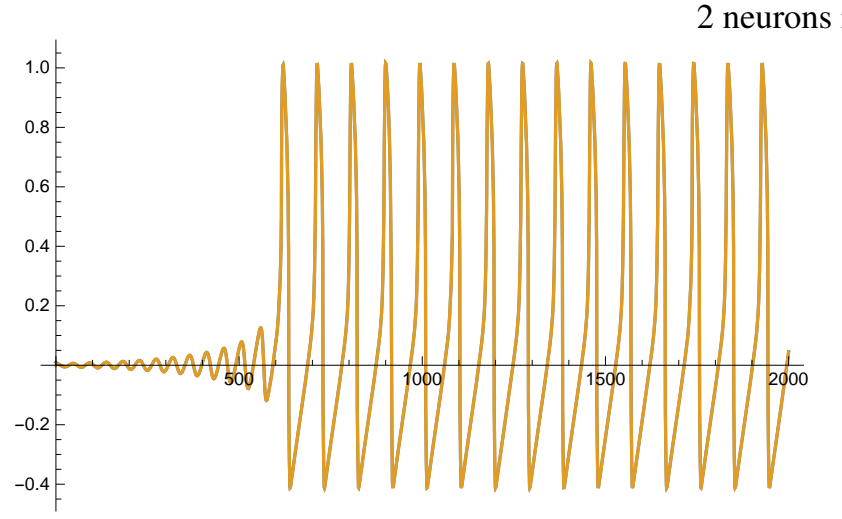

FIG. 2. $\tau=0$

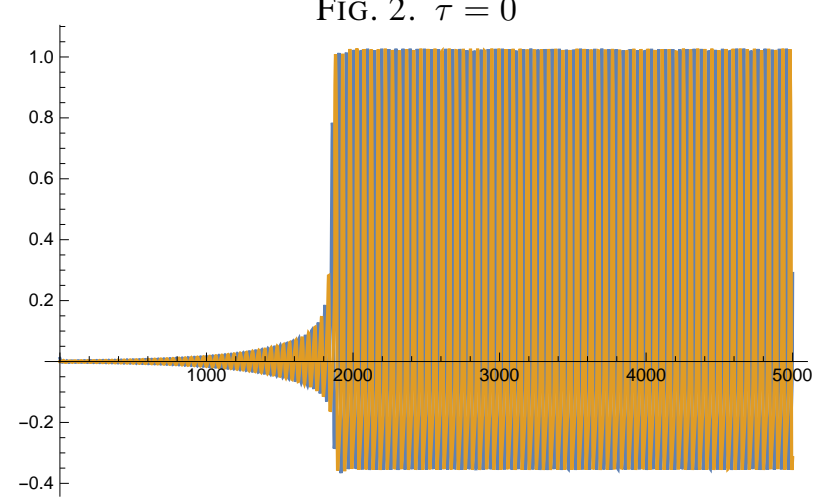

FIG. 4. $\tau=20$

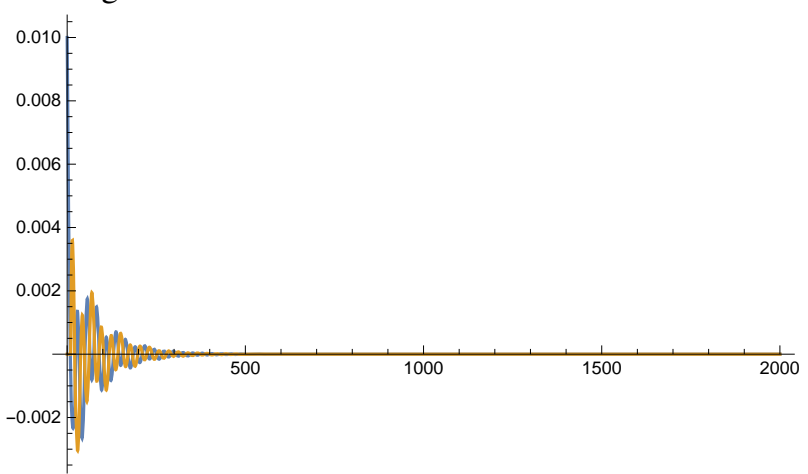

FIG. 3. $\tau=10$

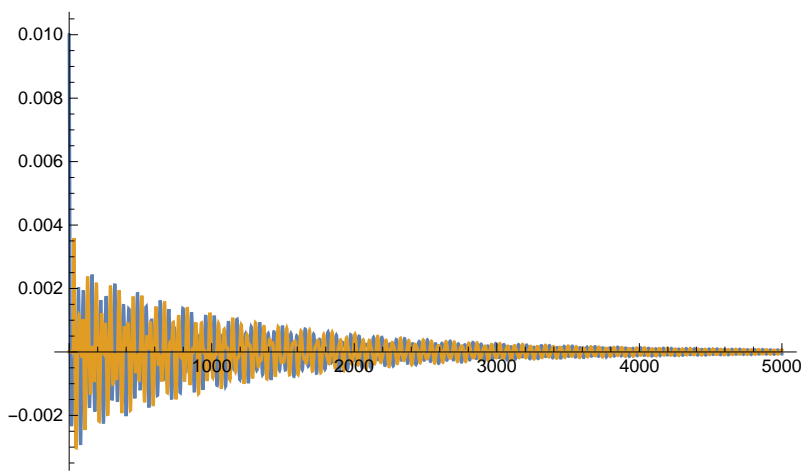

FIG. 5. $\tau=29$

3 neurons in a ring
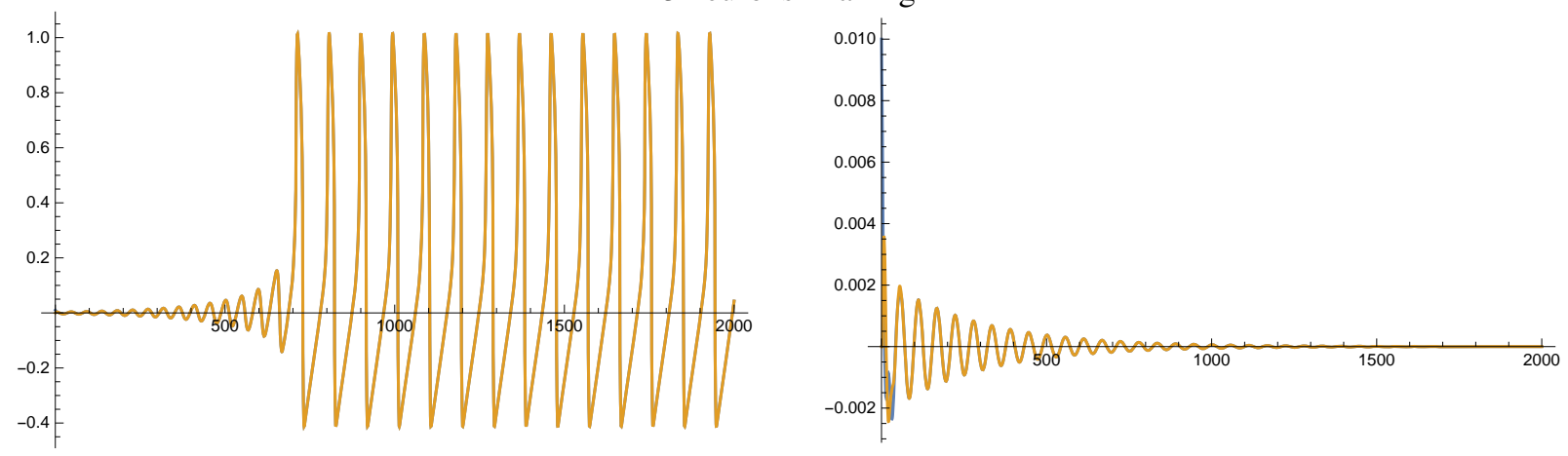

FIG. 6. $\tau=0$
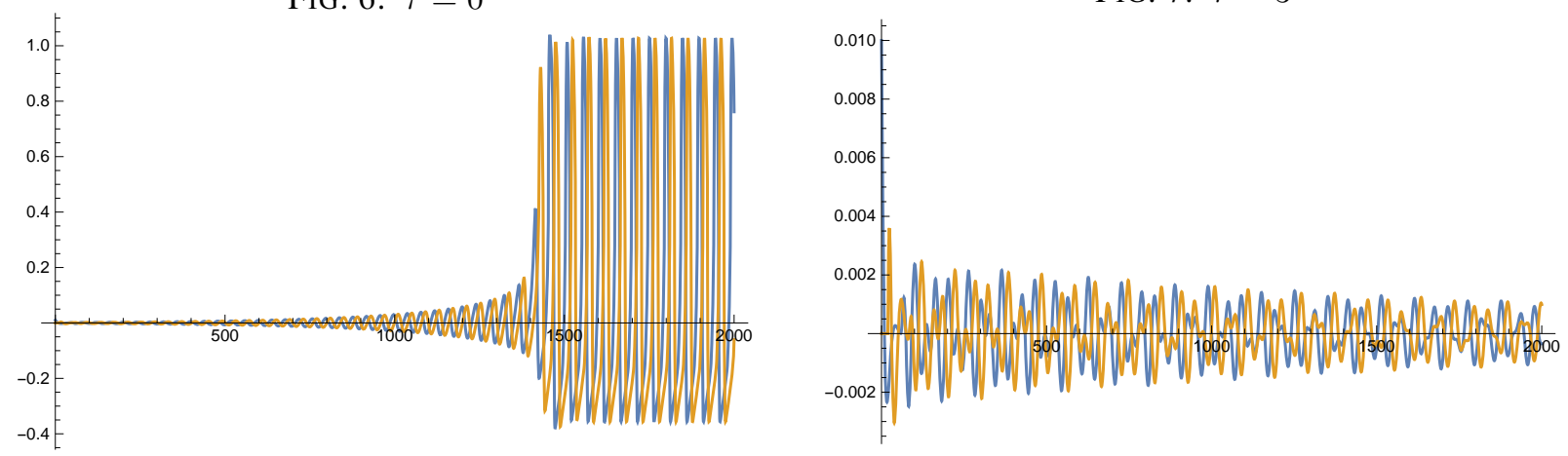

FIG. 8. $\tau=12$ 
3) A ring of four neurons. Then two series of candidates are $\tau_{1, k} \approx 1.70691+12.857505 k$ of the second kind, and $\tau_{2, k} \approx 5.983812+8.447756 k$ of the first kind. Then, for this unique equilibrium state, obtain two Hopf bifurcations: at $\tau \approx 1.70691$ - a transition from an unstable equilibrium to an asymptotically stable one (Fig. 10, 11), then at $\tau \approx 5.983812$ (Fig. 12).
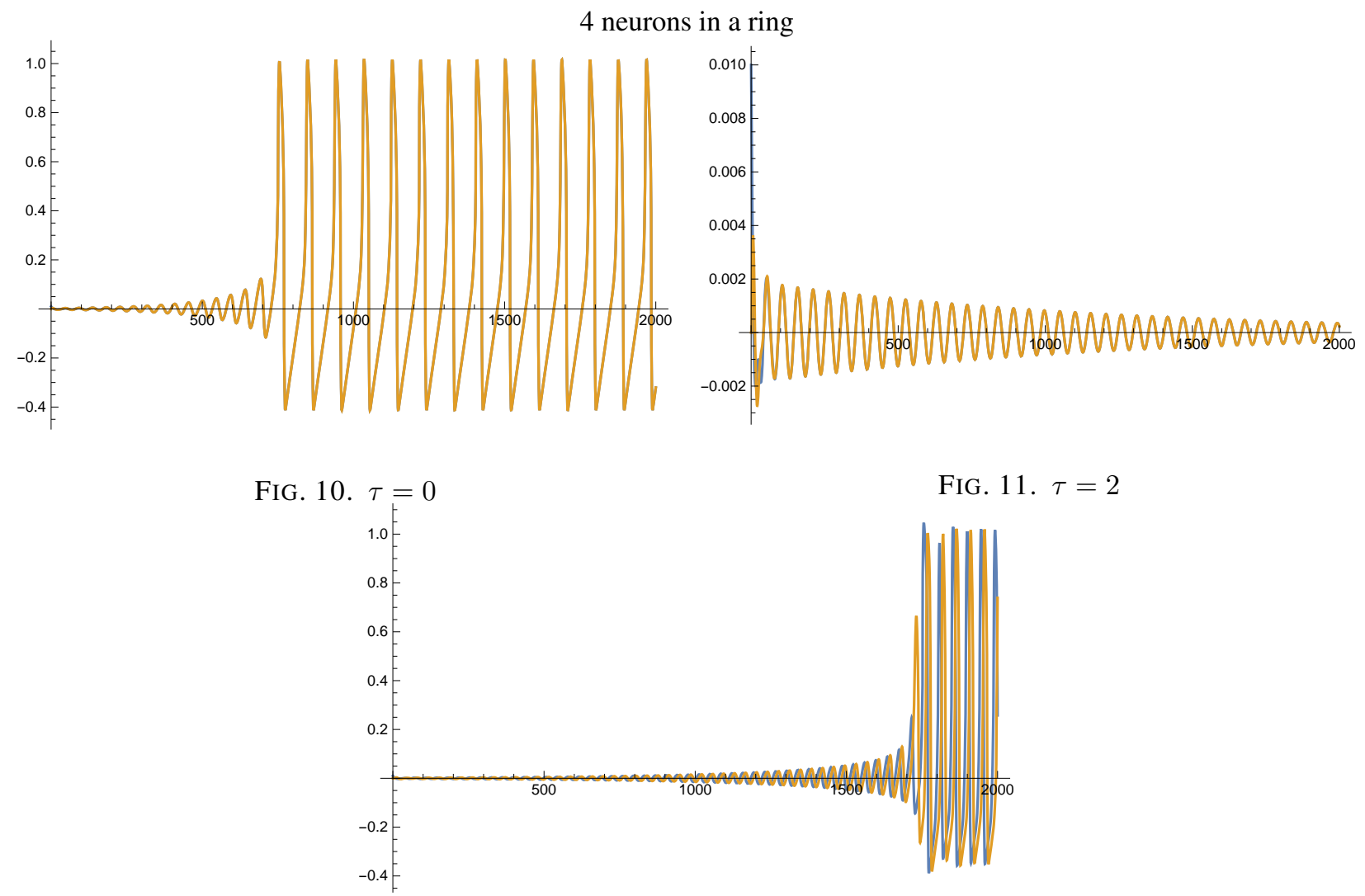

FIG. 12. $\tau=7$

\section{Conclusion}

In this work, a system in the form of an oriented ring of neurons was considered. Connection between the two neurons was delayed. To describe the behavior of one neuron, the FitzHugh-Nagumo model was used, and on its basis, the final model for the system in the form of a ring of neurons was built. For this system, the equilibrium states were determined, criteria for determining the stability of equilibrium states and, as a consequence, criteria for the presence of periodic solutions in the system were obtained. It has been shown that the period of self-oscillations in the ring of neurons does not have a linear dependence on the number of neurons in the ring. In this case, for a fixed number of neurons, the dynamics will be affected only by the total delay in the transmission of an impulse along the ring, and not by individual pairs of neurons. All results are then verified numerically.

\section{Acknowledgements}

This work was supported by the Russian Foundation for Basic Research (RFBR) project number 20-31-90036. 


\section{References}

[1] Schöll E., Hiller G., Hövel P., Dahlem M. Time-delayed feedback in neurosystems. Philosophical Transactions of the Royal Society A: Mathematical, Physical and Engineering Sciences. 2009. 1891 (367). 1079-1096.

[2] Burić N., Todorović D. Dynamics of FitzHugh-Nagumo excitable systems with delayed coupling. Physical Review E. 2003. 6 (67). 066222 p.

[3] Song Z., Xu J., Zhen B. Multitype activity coexistence in an inertial two-neuron system with multiple delays. International Journal of Bifurcation and Chaos. 2015. 13 (25). 1530040 p.

[4] Xu C., Zhang Q., Wu Y. Bifurcation analysis in a three-neuron artificial neural network model with distributed delays. Neural Processing Letters. 2016. 2 (44). 343-373.

[5] Karaoğlu E., Yılmaz E., Merdan H. Stability and bifurcation analysis of two-neuron network with discrete and distributed delays. Neurocomputing. 2016. 182. 102-110.

[6] Xu C. Local and global Hopf bifurcation analysis on simplified bidirectional associative memory neural networks with multiple delays. Mathematics and Computers in Simulation. 2018. 149. 69-90.

[7] FitzHugh R. Impulses and physiological states in theoretical models of nerve membrane. Biophysical journal. 1961. 6 (1). $445-466$.

[8] Nagumo J., Arimoto S., Yoshizawa S. An active pulse transmission line simulating nerve axon. Proceedings of the IRE. 1962. 10 (50). 20612070.

[9] Fedorov E.G., Popov A.I., Popov I.Y. Metric graph version of the FitzHugh-Nagumo model. Nanosystems: Physics, Chemistry, Mathematics. 2019. 10 (6). 623-626.

[10] Burić N., Grozdanović I., Vasović N. Type I vs. type II excitable systems with delayed coupling. Chaos, Solitons and Fractals. 2005. 5 (23). $1221-1233$. 